\title{
DIAGNÓSTICO E TRATAMENTO DA SÍNDROME DA ARDÊNCIA BUCAL
}

\author{
DIAGNOSIS AND TREATMENT OF ORAL BURNING SYNDROME
}

DIAGNÓSTICO Y TRATAMIENTO DE LA SÍNDROME DE ARDOR BUCAL

Recebido: 23/09/2013 Aprovado: 10/06/2015

\author{
Guilherme Henrique Borges ${ }^{1}$ \\ Marcelo Sivieri Araujo²
}

O objetivo desse estudo foi realizar uma revisão de literatura sobre os fatores associados ao diagnóstico e tratamento da síndrome de ardência bucal (SAB) que o cirurgião-dentista deve estar apto a reconhecer. As bases de dados consideradas foram: PUBMED, MEDLINE, LILACS e SCIELO no período de 2000 a 2014, com uso de descritores ligados a SAB. Foram encontrados 25 artigos e os resultados mostraram duas categorias - Diagnóstico do SAB e Tratamento do $S A B$. Verificaram-se diversas modalidades terapêuticas, porém estas são paliativas, na qual a associação de transtorno psicológico, depressão e ansiedade nos pacientes é um achado comum. Novos estudos são necessários para a elucidação da patogênese da SAB, a fim de proporcionar melhor qualidade de vida para os pacientes, e um maior conhecimento das modalidades de manejo e tratamento.

Descritores: Diagnóstico; Síndrome da ardência bucal; Terapêutica.

The objective of this study was to conduct a literature review on the factors associated with the diagnosis and treatment of burning mouth syndrome (SAB) that the dental surgeon should be able to recognize. The databases considered were: PUBMED, MEDLINE, LILACS and SCIELO in the period from 2000 to 2014, with use of descriptors connected to SAB. Were found 25 articles and the results showed two categories - Diagnosis of SAB and Treatment of $S A B$. Different therapeutic modalities exist, however these are palliative, where the association of psychological disorder, depression and anxiety in patients is a common finding. It is concluded that further studies are needed for the elucidation of the pathogenesis of the $\mathrm{SAB}$, in order to provide better quality of life for patients, and a greater awareness of the modalities of treatment.

Descriptors: Diagnosis; Burning mouth syndrome, Therapeutics.

El objetivo de este estudio fue realizar una revisión de literatura sobre los factores asociados con el diagnóstico y tratamiento del síndrome de ardor bucal (SAB) que el cirujano dentista debe ser capaz de reconocer. Las bases de datos consideradas fueran: PUBMED, MEDLINE, LILACS y SCIELO en el período de 2000 al 2014, con uso de descriptores involucrados con SAB. Fueron encontrados 25 artículos y los resultados mostraron dos categorías - Diagnóstico do $S A B$ y Tratamiento del SAB. Diversas modalidades terapéuticas existen, sin embargo éstos son paliativos, donde la asociación de trastorno psicológico, depresión y ansiedad en pacientes es un hallazgo frecuente. Nuevos estudios son necesarios, para la aclaración de la patogénesis de la SAB y, para ofrecer mejor calidad de vida para los pacientes y un mayor conocimiento de las modalidades de tratamiento.

Descriptores: Diagnóstico; Síndrome de boca ardiente; Terapéutica.

${ }^{1}$ Cirurgião Dentista. Especializando em Ortodontia pela Universidade Paulista. Mestrando em Ciências da Saúde pela Universidade Federal do Triângulo Mineiro (UFTM) guiodo@live.com.

${ }^{2}$ Cirurgião Dentista. Especialista em Patologia Bucal. Mestre em Odontologia. Doutor em Patologia Básica e Experimental. Professor da Universidade de Uberaba (UNIUBE) nos Departamento de Odontologia e Departamento de Fisioterapia. marcelo.sivieri@uniube.br. 


\section{INTRODUÇÃO}

$\mathrm{P}$ essoas com sintomas de dores, secura e sensação de ardência ou queimação na boca que procuram atendimento odontológico são tratados de diversas maneiras sem que se chegue a um diagnóstico ou prognóstico decisivo ${ }^{1}$.

A Síndrome da Ardência Bucal (SAB) é caracterizada por dor na cavidade bucal com ou sem sinais inflamatórios, mas sem lesões específicas $^{2-4}$. A sinonímia para SAB abrange glossodínia, distesia oral, glossopirose, glossalgia, estomatopirose, estomatodinia 5 .

A SAB é uma condição relativamente comum e ocorrendo mais em mulheres pósmenopausa e em pessoas idosas ou de meia idade, não havendo predomínio para etnia e classes socioeconômicas ${ }^{6}$.

A presença de dor na boca, alterações do paladar e da salivação, sem lesão clinicamente detectável na mucosa bucal, se caracterizam como uma tríade patognomônica para a SAB. A dor é do tipo queimação, de intensidade moderada a severa, podendo persistir por anos, acometendo as bordas laterais e ponta da língua. Pode haver sensação dolorosa em gengivas, lábios e mucosa jugal, sem alterações clínicas visíveis ao exame bucal e faríngeo ${ }^{4,5,7}$.

Nos pacientes acometidos pela $\mathrm{SAB}$, há aumento da sensação dolorosa no decorrer do dia, nos estados de tensão, fadiga, ao falar muito, à ingestão de alimentos picantes e quentes, ocorrendo melhora com a ingestão de alimentos frios, com a realização de algum tipo de trabalho e/ou distração. A sensação de queimação não segue a anatomia dos nervos periféricos e tipicamente acomete mais de um sítio 5 .

A queixa de lábios e boca seca, intensa salivação, hipogeusia e de disgeusia, e alteração no gosto principalmente para o metálico, salgado ou amargo são frequentemente encontrados nos pacientes com a Síndrome ${ }^{5,7}$. A composição salivar do paciente com a SAB pode apresentar alterações, como aumento na quantidade de potássio, proteínas e fosfatos, tornando a saliva mais espessa e pegajosa ${ }^{7}$.
A etiologia da SAB ainda é desconhecida, mas fatores locais, sistêmicos e psicogênicos são apontados como desencadeadores da doença, evidenciando assim, o seu caráter multifatorial ${ }^{7}$.

Os pacientes portadores da SAB procuram auxílio de especialistas como cirurgiões dentistas, otorrinolaringologista, dermatologista e fazem uso de corticóides, analgésicos, antibiótico, estrogênio, retinóides e psicotrópicos ${ }^{8}$.

A realização de anamnese, exame físico geral, exames da cavidade bucal e orofaringe minuciosos são de fundamentais para evitar que o tratamento seja baseado em tentativa e erro ${ }^{5}$.

Visando colaborar na ampliação do conhecimento dos profissionais da área da saúde, sobre os procedimentos úteis no diagnóstico e na escolha de modalidades terapêuticas da SAB, o objetivo desse trabalho foi realizar uma revisão de literatura sobre os fatores associados ao diagnóstico e tratamento da doença que o CirurgiãoDentista deve estar apto a reconhecer.

\section{MÉTODO}

Esta é uma revisão sobre publicações em revistas indexadas, acerca da síndrome de ardência bucal, considerando o período de 2000 a 2014, nos bancos de dados PUBMED, MEDLINE, LILACS e SCIELO.

Para a busca se utilizou os descritores: glossodynia, stomatodynia, etiopathogenesis, treatment, orofacial pain, burning mouth syndrome, neuropathicpain, oral phantompain, etiology, psychogenic factors, neuroinflammatory model, neuroplasticity, neuropathic theory, hormone disorders, drug-induced, antihypertensive drugs, angiotensin-converting enzyme inhibitors, angiotensin II receptor antagonists, clinical trials, clinical cases, antidepressants, analgesics, management, pathophysiology, evaluation, xerostomia.

Para a classificação quanto ao tema estudado, realizou-se uma leitura inicial, que originou categorias temáticas. 


\section{RESULTADOS}

Após a análise das publicações considerou-se 25 publicações, sendo: 5 monografias, 18 artigos científicos e dois capítulos de livros, que atendiam ao objetivo do estudo. 0 estudo apresentou duas categorias, a saber: Diagnóstico da $S A B$ e Tratamento da $S A B$

\section{DISCUSSÃO}

\section{Diagnóstico da $S A B$}

A SAB pode ser definida como uma entidade clínica caracterizada pela dor e/ou sensação de ardor localizada em determinada região ou estender por toda a mucosa bucal sem a detecção de lesões ou alterações do padrão da normalidade ${ }^{1-4,6,9,10}$. A patogenia desta doença ainda é desconhecida, o que dificulta o avanço na investigação de um tratamento eficaz ${ }^{3,11,12 .}$

Os portadores da SAB caracterizam-se por serem ansiosos, desconfiados, deprimidos, preocupados, socialmente isolados e com funções corporais e emocionais abaladas, com tendência de se cansarem com facilidade, sofrem de tensão muscular, costumam ter a voz monótona e são acometidos por possíveis palpitações e indigestão. Geralmente, são indivíduos hipocondríacos, cancerofóbicos, e que vivenciam ou passam por experiências estressantes. Podem apresentar graus de desordem mental e tendências neuróticas ${ }^{3,12}$.

Irritantes locais como alimentos condimentados, líquidos quentes, bebidas gasosas, café e chá fortes, certos sucos, dentifrícios e colutórios, são capazes de exacerbar o desconforto ${ }^{13}$. As disfunções do sistema mastigatório levam ao surgimento de hábitos parafuncionais, tais como o bruxismo, desencadeando a sensação de ardor na mucosa bucal ${ }^{4}$. 0 fumo, o álcool, o refluxo esofágico e próteses confeccionadas há anos e não adaptadas atuam como agentes irritantes sobre a mucosa bucal, ressecando-a e causando sensação de desconforto ${ }^{12}$.

Um importante quadro agravante da SAB é a xerostomia, como consequência da diminuição da função das glândulas salivares, com o avanço da idade, e o efeito colateral do uso de medicamentos como os antiespasmódicos, antidepressivos, antipsicóticos, relaxantes músculoesqueléticos, antiparkinsonianos, antiarrítmicos, anti-histamínicos, anticonvulsivantes, ansiolíticos, benzodiazepínicos, moderadores de apetite, diuréticos e anti-hipertensivos ${ }^{4,12,13}$.

Em pacientes com SAB foram documentadas alterações salivares significativas no potencial hidrogeniônico $(\mathrm{pH})$, capacidade tampão, proteínas, mucina e imunoglobulinas. As alterações nos constituintes salivares parecem ter significado na Síndrome, em se tratando da redução geral do fluxo salivar, e sugerem envolvimento da função simpática e parassimpática, além da injúria neuropática no desenvolvimento da doença ${ }^{7,4}$.

Acredita-se que a SAB possa ser resultante de doenças sistêmicas específicas ou deficiências nutricionais, incluindo vitaminas B e ferro. Entretanto, ainda não foi encontrada relação consistente, já que o tratamento e a correção dos achados, não diminuem a queimação bucal e outras queixas associadas ${ }^{5}$.

Uma maior incidência de lesões de tecidos moles da boca, como gengivite, periodontite, lesões ulceradas ou erosivas, ou língua geográfica, fissurada, festonada ou eritematosa, síndrome de Sjogren, outras doenças do tecido conjuntivo e o Diabete mellitus tem sido relatada nos pacientes com SBA, e a possibilidade das condições causarem alterações neuropáticas irreversíveis não foi completamente explorada ${ }^{11}$.

A SAB pode ser uma condição neuropática mediada centralmente ou perifericamente com etiologias diversas. Considerando o aumento da queimação bucal após o bochecho com anestésico tópico, tem sido sugerido que a queimação bucal pode ser uma condição neuropática central que resulta na diminuição da inibição periférica do nervo trigêmeo ${ }^{11}$. As alterações do paladar e tolerância à dor pelo calor são notadas nos pacientes com $\mathrm{SAB}$, envolvendo a perda de função das fibras nervosas de pequeno calibre $^{11}$. 0 mecanismo pelo qual o sistema nervoso é envolvido na $S A B$ permanece pouco explorado e conhecido ${ }^{4,7}$. 
$\mathrm{Na} \mathrm{SAB}$, há relatos de casos de queimação bucal decorrentes do uso de inibidores da enzima conversora de angiotensina (ECA), como o captopril, maleato de enalapril e lisinopril, que sofreram remissão após a descontinuação da medicação ${ }^{14}$. A perda da sensação do paladar é relatada com o uso de inibidores da ECA, sugerindo mais uma ligação entre a dor, o paladar e a $\mathrm{SAB}^{7,12}$.

A SAB é mais prevalente em mulheres após a menopausa, com dados epidemiológicos sugerindo um acometimento equilibrado entre homens e mulheres ${ }^{15}$. Se a menopausa pode ser considerada como um fator relacionado para com a síndrome, seu mecanismo ainda não está claro, pois se sugere que a queimação bucal, geralmente, não é revertida com o tratamento por reposição hormonal7.

A caracterização da síndrome pode iniciar pela queixa de ardência constante na boca sem qualquer lesão detectada ao exame físico da cavidade bucal $^{16}$. Os pacientes relatam a sensação de boca queimada, que inicia pela manhã e, na maioria dos casos, tende a intensificar-se no decorrer do dia. A presença do sintoma de ardência pode estar associada a doenças e/ou alterações sistêmicas, entre elas a síndrome de Sjögren, radioterapia de cabeça pescoço e alterações hormonais ${ }^{7}$.

0 diagnóstico da SAB é eminentemente clínico. A presença de qualquer lesão, a hipótese da síndrome estará descartada. Durante o exame clínico, as estruturas bucais, devem ser verificadas e, se estão dentro dos padrões de normalidade,tentando localizar a origem da dor. Além da anamnese detalhada, procura-se conhecer o estado emocional da pessoa.

Como recursos diagnósticos complementares, os exames micológicos, como cultura para fungos, exames hematológicos, níveis de ferro sérico, vitamina B12, dosagem hormonal, secreção salivar e os testes sorológicos para anticorpos da síndrome de Sjogren, podem ser utilizados. É aconselhável, antes de estabelecer o diagnóstico final de SAB que o cirurgião dentista, deva discutir com outro profissional de saúde especialista e conversar com familiares ${ }^{17}$.

\section{Tratamento da $S A B$}

O desconhecimento de uma causa específica para a SAB e a multiplicidade de possíveis fatores etiológicos associados à condição, tornam o manejo clínico do paciente uma tarefa desanimadora ${ }^{12}$.

Busca-se na SAB empregar medidas paliativas que visam eliminar fatores, locais ou gerais, que agravam a sintomatologia. Essas medidas incluem, entre outras, o uso de saliva artificial, a estimulação do fluxo salivar, ajuste de próteses, remoção de cálculo dentário e orientação de higiene oral ${ }^{11}$. A condição psíquica não pode ser menosprezada, constituindo uma orientação importante, o encaminhamento para avaliação psicológica ou psiquiátrica ${ }^{12}$.

Nos pacientes com SAB há significante incidência de espécies de Cândida, onde a remissão dos sintomas para a síndrome ocorre depois de instituída terapia antifúngica. Para indivíduos com deficiência de vitaminas do complexo $B$ e que apresentam a $S A B$, a terapia de reposição se mostrou efetiva. $\mathrm{Na}$ xerostomia associada a $\mathrm{SAB}, \quad$ o uso de saliva artificial, géis hidratantes, enxagüatórios bucais, aplicação tópica de flúor e sialagogos contribuíram em estudos para a remissão dos sintomas da síndrome ${ }^{18}$.

$\mathrm{Na}$ ausência de fatores que possam explicar a sintomatologia apresentada na SAB uma terapia farmacológica tem sido recomendada para aliviar o incômodo na doença. 0 uso de analgésicos, corticosteróides, sialagogos, antidepressivos, benzodiazepínicos, e anticonvulsivantes pode ser uma boa opção antes da definição do diagnóstico da $\mathrm{SAB}^{4,18,19}$.

A principal razão para o insucesso no tratamento da SAB parece ser o fracasso no acesso aos fatores associados ${ }^{5}$, como no caso de pacientes com deficiência de lítio sérico que desenvolvem a $\mathrm{SAB}$, cuja reposição faz desaparecer os sintomas ${ }^{12}$.

A SAB tem sido tratada com antidepressivos tricíclicos (TCA) em baixa dosagem, com base nos relatos iniciais da efetividade como analgésicos que aliviam a 
queimação bucal ${ }^{11}$. Muitos tricíclicos têm sido usados, incluindo amitriptilina, desipramina, nortriptilina, imipramina e clomipramina, embora apenas a amitriptilina tenha sido avaliada em experimentos clínicos controlados ${ }^{7}$.

Estudos sugerem que os benzodiazepínicos, incluindo o clonazepam, podem ser eficazes na $\mathrm{SAB}^{7}$. 0 clonazepam tem se mostrado eficaz atenuante da disgeusia pelo paladar e ressecamento bucal somados à queimação bucal presentes na $\mathrm{SAB}^{7}$.

Outros medicamentos e tratamentos recomendados para o alívio sintomático da queimação bucal presente na $S A B$, incluem a capsaicina tópica; sulfato de tranilcipromina, inibidor da monoamina oxidase em combinação com diazepam; além do anestésico sistêmico mexiletina, um bloqueador dos canais de sódio usodependente, sendo indicados para condições neuropáticas dolorosas ${ }^{3,7}$.

Os tratamentos para a SAB mostram-se por vezes empíricos e, apesar de na maioria das vezes, se basearem em protocolos clínicos descritos na literatura. Uma vez descartada a atuação de fatores locais, o tratamento da síndrome, deverá ser realizado como se o profissional estivesse diante de uma dor do tipo neuropática ${ }^{20,21}$.

A história da dor na SAB é muito importante como fator colaborador no diagnóstico da síndrome. Esta deve ser avaliada quanto à duração, intensidade, local, fatores de alívio ou agravamento, presença de salivação abundante ou xerostomia, tipo de alimentação, alterações na gustação, uso de enxaguantes bucais, tipo de creme dental, hábitos como uso de goma de mascar, cigarro, bebidas alcoólicas, relação da dor com o uso de aparelhos protéticos, presença de movimentos para-funcionais, medicação utilizada diariamente e a história psicológica ${ }^{22,23}$.

Devido a dificuldade de se encontrar uma maneira definitiva e eficaz de tratamento podem ser sugeridos os seguintes esquemas terapêuticos ${ }^{2-4,11}$ :

\section{- Tratamento local ou tópico}

Lidocaína 2\% (uso tópico): reduz a sintomatologia dolorosa sem causar aumento da sensação de ardor no início;

Capsaicina tópica a 0,025\% e 0,075\%: neuropeptídeo natural, obtido de plantas da família das solanáceas, que produz uma efetiva analgesia seletiva em síndromes de dor localizada, quando usada como droga única ou associada a medicamentos por via oral. Deve ser utilizada duas vezes ao dia, pela manhã e a tarde, durante três semanas;

Laserterapia: alternativa analgésica, provavelmente devido ao aumento da produção de $\beta$-endorfinas e serotonina, no controle de prostanglandina e na bioestimulaçao das fibras musculares.

\section{- Tratamento sistêmico}

Ansiolíticos benzodiazepínicos: deve ser usado na dose inicial de $0,25 \mathrm{mg} /$ dia, podendo atingir $3 \mathrm{mg} / \mathrm{dia}$, até que os sintomas diminuam sem que ocorram efeitos secundários do medicamento;

Antidepressivos tricíclicos (amitriptilina, nortriptilina, clomipramina): usar dose inicial de $10 \mathrm{mg} /$ dia, podendo ser aumentada em 10 mg a cada dia, até 40 a $70 \mathrm{mg} /$ dia, dividida em três doses diárias, usar até a redução dos sintomas ou surgimento de efeitos colaterais; a dose máxima de ser de $150 \mathrm{mg} /$ dia;

Antidepressivos inibidores da serotonina: utilizar $50 \mathrm{mg} /$ dia durante 2 semanas; e depois, $150 \mathrm{mg} /$ dia até o desaparecimento dos sintomas;

Estabilizadores do humor (lítio): usar 0,4 a 1,2 $\mathrm{g} /$ dia até a reposição dos níveis séricos de 0,60 a $1,20 \mathrm{mEq} / \mathrm{L}$;

Anticonvulsivantes: a dose inicial de ser de $100 \mathrm{mg} /$ dia, aumentando em $100 \mathrm{mg} /$ dia a cada 4 dias ou 7 dias, fracionadas em 3 vezes ao dia, até a remissão dos sintomas ou surgimento de efeitos colaterais;

Antiespasmódicos com ação nos músculos lisos (clordiazepóxido): utilizar $5 \mathrm{mg} /$ noite, aumentando em $5 \mathrm{mg}$ a cada 4 dias ou 7 dias, 3 vezes ao dia, até a diminuição dos sintomas ou aparecimento de efeitos colaterais;

Ácido alfa lipóico: utilizar a dose de 800 $\mathrm{mg} /$ dia, durante 8 semanas até a redução dos sintomas. 
A indicação de psicoterapia também tem sido sugerida para o tratamento da SBA e podem trazer benefícios para os pacientes, principalmente aqueles que se apresentam ansiosos, deprimidos, tensos e os cancerofóbicos $3,21,23,24,25$.

\section{CONCLUSÃO}

Embora os possíveis fatores etiológicos tenham sido apontados para a síndrome, sua causa específica permanece desconhecida e, consequentemente, não há tratamento eficaz. Sugere-se que a SBA possa ser resultante de doenças sistêmicas específicas como a síndrome de Sjogren, alterações sistêmicas como a xerostomia, disgeusia, radioterapia de cabeça e pescoço, alterações hormonais e alterações neuropáticas, e deficiências nutricionais, incluindo vitaminas B e ferro.

Os mecanismos patogênicos da SBA permaneçam desconhecidos. Verifica-se portanto, que a doença está intimamente relacionada com alterações psicológicas, ainda não comprovadas cientificamente.

0 desconhecimento de uma causa específica para a síndrome e a multiplicidade de possíveis fatores etiológicos associados à condição tornam o manejo clínico desses pacientes uma tarefa desafiadora. 0 cirurgião dentista deve estar preparado para reconhecer as várias manifestações de sinais e sintomas da $\mathrm{SAB}$, podendo assim, buscar a melhor modalidade terapêutica em parceria com outras especialidades da área da saúde, a fim de ajudar o paciente que apresenta a síndrome.

Novos estudos fazem-se necessários a fim de que, se elucide a causa da síndrome para proporcionar melhor qualidade de vida aos pacientes com SAB.

\section{REFERÊNCIAS}

1. Salort-Llorca C, Mínguez-Serra MP, Silvestre FJ. Drug-induced burning mouth syndrome: a new etiological diagnosis. Med Oral Patol Oral Cir Bucal. 2008; 13(3):167-70. 2. Souza FT, Santos TP, Bernardes VF, Teixeira AL, Kummer AM, Silva TA, et al. The impact of burning mouth syndrome on health-related quality of life. Health Qual Life Outcomes. 2011; 9:57.
3. Campillo ERR, López, JL. Evaluation of the response to treatment and clinical evolution in patients with burning mouth syndrome. Med Oral Patol Oral Cir Bucal. 2013; 18(3): 403-10.

4. Silva LA, Siqueira JTT, Teixeira MJ, Siqueira SRDT. The role of xerostomy in burning mouth syndrome: a case-control study. Arq Neuropsiquiatr. 2014; 72(2):91- 8.

5. Cerchiari DP, Moricz RD, Sanjar FA, Rapoport PB, Moretti G, Guerra MM. Síndrome da boca ardente: etiologia. Rev Bras Otorrinolaringol. 2006; 72(3):419-24.

6. Oliveira GMR, Pereira HSC, Silva-Júnior GO, Picciani BLS, Ramos RT, Pestana SG, et al. Síndrome da ardência bucal: aspectos clínicos e tratamento. Rev Hosp Univ Pedro Ernesto. 2013; 12(1):21-9.

7. Grushka M, Epstein JB, Kawalec JS. Síndrome da ardência bucal. In: Silverman Jr. $S$, Eversole LR, Truelove EL, (Orgs). Fundamentos de medicina oral. Moreira LC, tradutor. Rio de Janeiro: Guanabara Koogan; 2004. p.349-52.

8. Gonçalo CS, Castro CM, Bonon MM, Motta PMR, Dahdal AB, Batista J. Planejamento e execução de revisões sistemáticas da literatura. Bras Med. 2012; 49(2):104-10.

9. Maidana JD, Cherubini K, Krapf SMR. Associação entre síndrome da ardência bucal e hipossialia. RFO UPF. 2005; 10(1):24-8.

10. Mock D, Chugh D. Burning mouth syndrome. Int J Oral Sci. 2010; 2(1):1-4.

11. Monteiro AD, Sousa ACV, Matos FZ, Carvalhosa AA, França DCC, Aguiar SMHCA. Síndrome da ardência bucal, correlações fisiopatológicas, medicamentosas: revisão de literatura. Rev Odontol de Araçatuba. 2011; 32(2):33-7.

12. Cherubini K, Maidana JD, Weigert KL, Figueiredo MA. Síndrome de ardência bucal: revisão de cem casos. Rev Odonto Ciênc. 2005; 20(48):109-13.

13. Mercadante $S$, Calderone L, Villari $P$, Serretta L, Sapio M, Casuccio A, et al. The use of pilocarpine in opioid-induced xerostomia. Palliat Med. 2000; 14(6):529-31.

14. López-Jornet P, Camacho-Alonso F, Andujar-Mateos P, Sánchez-Siles M, GómezGarcía F. Burning mouth syndrome: Update. 
Med Oral Patol Oral Cir Bucal. 2010; 15(4): 562-8.

15. Frutos R, Rodríguez $\mathrm{S}$, Miralles $\mathrm{L}$. Machuca G. Manifestaciones orales y manejo odontológico durante la menopausia. Med Oral. 2002; 7:26-35.

16. Minguez-Sanz MP, Salort-Llorca C, Silvestre-Donat FJ. Etiology of burning mouth syndrome: a review and update. Med Oral Patol Oral Cir Bucal. 2011; 16(2):144-8.

17. Klasser GD, Fischer DJ, Epstein JB. Burning mouth syndrome; recognition, understanding and management. Oral Maxillofac Surg Clin North Am. 2008; 20(2):255-71.

18. Studart-Soares EC, Costa FWG, Fontenele B. Síndrome de ardência bucal e relato de casos. Odontol Clín Cient. 2007; 6(3):259-62.

19. White TL, Kent PF, Kurtz DB, Emko P. Effectiveness of gabapentin for treatment of burning mouth syndrome. Arch Otolaryngol Head Neck Surg. 2004; 130:786-88.

20. Lopez-Jornet P, Camacho-Alnso F, LeonEspinosa S. Síndrome de ardência bucal, hábitos parafuncionais oral e perfil psicológico de um estudo de caso longitudinal. J Eur Acad Dermatol Venereol. 2009; 23(3):363-5.
21. Steele JC, Bruice AJ, Drage LA, Rogers RS. Alpha-lipoic acid treatment of 31 patientes with sore, burning mouth. Oral Dis. 2008; 14:529-532.

22. López- Jornet $\mathrm{P}$, Camacho - Alonso F, Andujar - Mateos P, Sánches - Siles M, Gómez - Garcia F. Burning syndrome: an update. Med Oral Patol Oral Cir Bucal. 2010; 15(4):E562-8.

23. Grushka M, Ching V, Epstein J. Síndrome de ardência bucal. Adv Otorhinolaryngol. 2006; 63:278-87.

24. Minguez Serra MP, Salort Llorca C, Silvestre Donat FI. O tratamento farmacológico da síndrome de ardência bucal: uma revisão e atualização. Med Oral Patol Oral Cir Bucal. 2008, 12(4):299-304.

25. Alan R, Hirsch MD, Ziad A. Pilot study: alleviation of pain in burning mouth syndrome with topical sucralose. Headache. 2011, 51(3):444-6.

\section{CONTRIBUIÇÕES}

Guilherme Henrique Borges participou da concepção, delineamento, análise dos artigos e redação do artigo. Marcelo Sivieri Araujo contribuiu no delineamento e revisão crítica. 